\title{
First-Fault Detection in DC Distribution with IT Grounding based on Sliding Discrete Fourier-Transform
}

\author{
Rafael Peña-Alzola, Senior Member, IEEE, Michal Sztykiel, Catherine E. Jones, Patrick J. Norman, Gareth Moore \\ Josep Pou, Fellow, IEEE, and Graeme M. Burt, Member, IEEE,
}

\begin{abstract}
Dc distribution minimises the number of power conversion stages and hence it lowers the overall cost, power losses and weight of a power system. Critical systems use IT grounding because it is tolerant to the first-fault. Hence, this is an attractive option for hybrid electric aircraft (HEA), which combines gas engines with electric motors driven by power electronic converters. This letter proposes an accurate implementation for the procedure of first-fault detection with IT grounding. Ac component injection along with the Sliding Discrete Fourier Transform (SDFT) is used to estimate the fault impedance. The procedure is very accurate due to the heavy filtering of the implicit moving average filter (MAF). Further computation savings are obtained by using the double look-up tables and the Goertzel algorithm for the SDFT. Results are validated by simulations and experiments.
\end{abstract}

Index Terms-Hybrid electric aircraft, Grounding, Permanent insulation monitor, IT systems, Sliding Discrete Fourier Transform, Moving average filter, Goertzel Algorithm.

\section{INTRODUCTION}

$\mathbf{H}$ HYBRID electric aircraft (HEA) has been proposed in order to reduce fuel-consumption, emissions and noise [1]. The thrust in HEA is provided by the gas engine and electric motor driven fans. DC distribution is an interesting option for HEA as it avoids double conversions and therefore lower costs, power losses and weight. Grounding in aircraft is usually TN-C [2], with the ground connection and the return path sharing the same cable to reduce weight. Near the passengers, TN-C-S is used for extra safety with the ground and the neutral being separate cables. For an aeroplane with aluminium fuselage, the aeroplane structure forms the ground plane [3]. In state-of-the-art aeroplanes, more than $50 \%$ of the structure is made of composite materials such as carbon fibre reinforced polymers (CFRP) with superior mechanical performance and lighter weight. However, the comparatively poor electrical conductance of CFRP compared with metals has resulted in the need to redesign the return/ground path [4], [5] using Current Return Networks (CRN) or Electrical Structural Networks (ESN). In CRN, the return/ground cables

This work was supported by the Rolls-Royce University Technology Centre Program at the University of Strathclyde, Glasgow.

R. Peña-Alzola, C. E. Jones, P. J. Norman and G. M. Burt are with the University of Strathclyde, Glasgow G1 1RD. Scotland, United Kingdom (email: \{rafael.pena-alzola, catherine.jones, graeme.burt\}@ strath.ac.uk).

M. Sztykiel was with the University of Strathclyde, Glasgow and now he is with Woodford Engineering Consultancy WEC UK Ltd, Bristol, BS34 7PZ, United Kingdom. (e-mail: michal.sztykiel@wecuk.com).

G. Moore is with the Rolls-Royce Electrical, Derby, DE24 8BJ, United Kingdom (e-mail: gareth.moore@ rolls-royce.com).

J. Pou is with the Nanyang Technological University, Singapore 639798 (e-mail: j.pou@ntu.edu.sg).

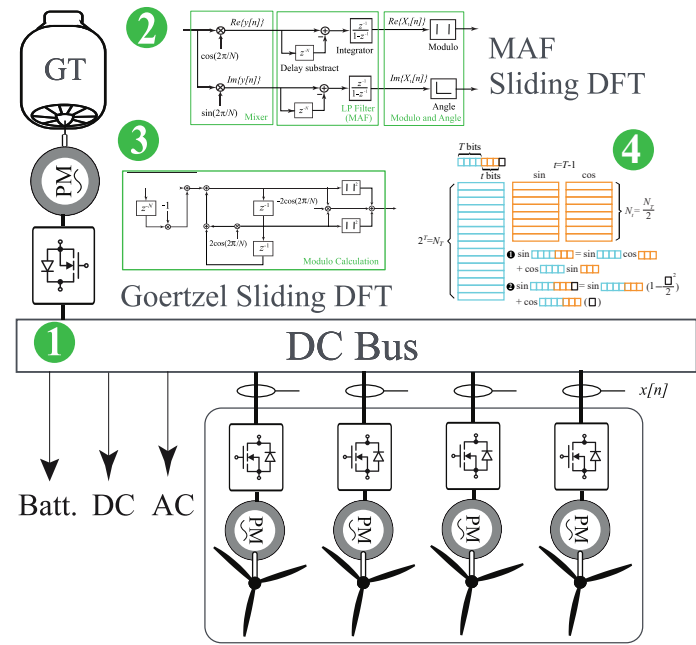

Fig. 1. Conceptual representation of the proposals of the letter. 1) example of HEA using IT grounding, 2) ac injection and sliding Fourier transform, 3) Goertzel implementation of SDFT and, 4) efficient implementation of double look-up table.

are deployed along the fuselage and in ESN, the metallic frame of the aircraft is used as the ground plane. In contrast to a TN-C or TN-C-S systems that result in high fault-currents, ungrounded systems (IT grounding) allows service continuity for the first fault. Hence, IT grounding is used in surgery rooms and telecom systems [6]. IT grounding is also routinely used in marine electrical systems [7] and can help to reduce the weight of the return cables. The service continuity makes IT grounding an attractive option for the HEA, where the sudden loss of thrust from an electric motor driven fan could lead to a potentially dangerous situation. As soon as the first fault occurs in an ungrounded system, it should be detected by the permanent insulation monitor (PIM) and take the necessary actions (e.g. shut down the faulty electric motor/generators gradually). After the first fault in IT grounding, the system becomes effectively grounded.

Passive techniques for PIM such as the method of the voltmeter balancer [6] are based on simple measurements. Passive techniques cannot detect symmetrical faults that occur if the insulation on all conductors degrades symmetrically with resistances dropping to an equal degree (for example due to moisture). In order to overcome this difficulty, active techniques that inject signals to estimate the fault impedance [8] are required. The abundance of power electronic converters has an impact on IT systems because their common mode 


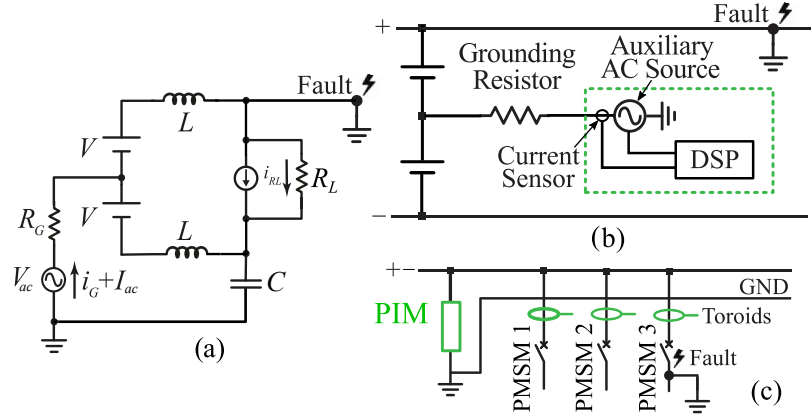

Fig. 2. (a) First-fault detection by using an auxiliary ac voltage source, (b) Permanent insulation monitoring (PIM), and (c) Fault tracking by using an ac current source.

EMI filters have capacitors connected to ground (Class-Y safety capacitors designed to fail open). This provides a path to ground during faults, which can lead to dangerous touch voltages [7]. For these reasons, the current leakage through the Y-caps is limited [7], [9]. Moreover, the AC path of the capacitance to ground makes difficult the permanent insulation monitoring. Resistive and capacitive components of the fault impedance are determined by using Fourier analysis such as synchronous demodulation (lock-in amplifier) [10] or fast Fourier transform (FFT) [11]. These procedures require fast and accurate calculations for the sine and cosine functions, for example using two-level look-up tables [12]-[15]. Ac signals are also used to trace the fault by placing toroids at the loads. The commutation method can also be used for PIM [16].

Injecting ac signals with Fourier analysis has been used for estimating the impedance in ac and dc distribution. Ref. [17] uses direct implementation of the DFT, while [18] uses the Goertzel algorithm to reduce the computations. The technique shown in [19] by Paz and Ordonez is particularly interesting as it uses a lock-in amplifier [20] combined with the heavy filtering properties of the moving average filter (MAF) [21]. This technique has been successfully used in land-based DC microgrid [19], [22]-[25] and solar applications [26], [27] but never in IT grounding for aircraft. Considerations on its relation to the sliding discrete Fourier Transform (SDFT) were not explained in these references.

This letter proposes a practical implementation, summarized in Fig. 1, for detecting the first-fault with ac injection and the SDFT. Section II explains the procedure based on the SDFT, which has an implicit MAF. Section III shows an efficient implementation based on the double look-up table. Section IV explains the Goertzel implementation of the SDFT. Simulation and experimental results are shown in Section V and VI, respectively. Finally, the letter is concluded in Section VII.

\section{First-Fault Detection Procedure with IT GROUNDING}

Fig. 2(a) shows the procedure that injects ac current with angular frequency $\omega_{a c}$ in order to detect the fault by estimating its impedance. There is an auxiliary ac voltage source in series with the ground resistance. After measuring the ac component of the ground current, the fault resistance $R_{F}$ can be estimated as follows:

$$
\frac{V_{a c}}{I_{a c}}=R_{G}+\left(R_{F}+j L \omega_{a c}\right) \|\left(j L \omega_{a c}+\frac{1}{j C \omega_{a c}}\right)
$$

assuming a very large $R_{L}$. The configuration of the PIM is shown in Fig. 2(b). Once the fault is detected, current sensors are distributed in the different permanent magnets synchronous motors (PMSM) to locate it as shown in Fig. 2(c). The ground fault is evidenced by detecting a current component with the selected angular frequency $\omega_{a c}$.

\section{A. Impedance Estimation}

The straightforward approach to measure the fault impedance is using the short-time discrete Fourier transform. The different spectral components are calculated within a time window $w[n]$ as follows:

$$
X_{k}[n]=\sum_{m=-\infty}^{\infty} x[n] w[k-m] e^{-j \frac{2 \pi k}{N}}
$$

The period of the injected ac current is $T_{a c}=2 \pi / \omega_{a c}$. If a rectangular window with the same duration as $T_{a c}$ is considered, this corresponds to $N$ discrete samples so that $N T_{s}=T_{a c}$ with $T_{s}$ the sampling period; this results in $N=2 \pi /\left(T_{s} \omega_{a c}\right)$. Eq. (2) can be expressed as follows:

$$
X_{k}[n]=\sum_{m=n-N}^{n-1} x[m] e^{-j \frac{2 \pi k m}{N}}
$$

This formulation includes an extra sample as a computation delay. The fundamental component $k=1$ is equal to:

$$
X_{1}[n]=\sum_{m=n-N}^{n-1} x[m] e^{-j \frac{2 \pi m}{N}}
$$

Fig. 3(a) shows the block diagram of the SDFT implementation. Defining the complex sequence illustrated in Fig. 3(b),

$$
y[n]=x[n] \cos \left(\frac{2 \pi}{N} n\right)-j x[n] \sin \left(\frac{2 \pi}{N} n\right)
$$

as resulting from the mixer of a lock-in amplifier, the fundamental component can be calculated by passing the previous sequence $y[n]$ through an MAF:

$$
X_{1}[n]=\sum_{m=n-N}^{n-1} y[m]
$$

The MAF can be expressed as two delayed integrators as:

$$
X_{1}[n]=\sum_{m=0}^{n-1} y[m]-\sum_{m=0}^{n-N-1} y[m]=\sum_{m=0}^{n-1}(y[m]-y[m-N])
$$

In the z-domain, this is equivalent to (see Fig. 3(b)):

$$
X_{1}(z)=\left(1-z^{-N}\right) \frac{z^{-1}}{1-z^{-1}} y(z)
$$




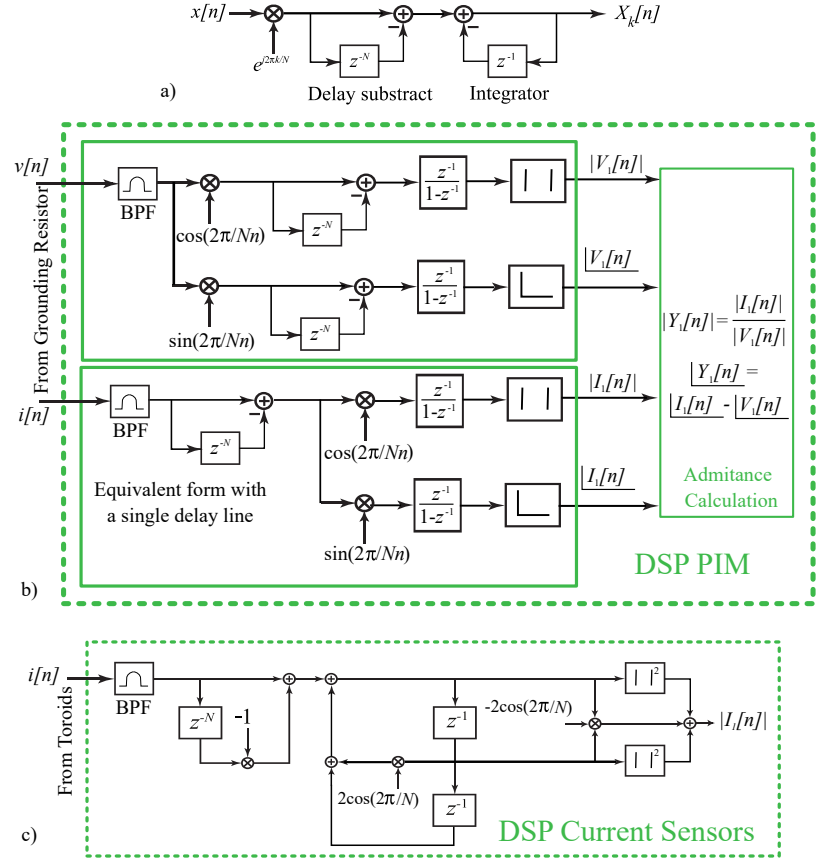

Fig. 3. (a) Short-time DFT, (b) sliding DFT for the fundamental component $k=1$ equivalent to a lock-in amplifier with MAF as a lowpass filter, (c) DSP procedure for the current sensor to locate the ground-fault using Goertzel algorithm.

Hence, the lock-in amplifier using an MAF as a low pass filter is equivalent to the sliding DFT for the fundamental component. The response delay of the MAF is:

$$
\tau_{M A F}=N T_{s} / 2=\pi / \omega_{a c}
$$

It can be seen that $\omega_{a c}$ determines the PIM detection speed and $T_{s}$ should be sufficiently small for accurate frequency discrimination with the mainlobe width $4 \pi /(N+1)$ [11] of the rectangular window. Fig. 3(b) shows the calculation of the amplitudes and angles of the current and voltage signals and hence the conductance and susceptance. One of the forms for the MAF in Fig. 3(b) is more computationally efficient by using only a single delay.

\section{EFFICIENT IMPLEMENTATION OF DOUBLE LOOK-UP TABLES}

The calculations for the sine and cosine functions use a double look-up table with optimized size. Because of symmetries of the sine and cosine functions, only one quarter of the waveform is necessary for the main table. The resolution of the main table is $\Delta_{T}=90^{\circ} / N_{T}$, with $N_{T}$ the number of data points. One entry of the main table is subdivided in $N_{t}$ intervals and two tables (for the sine and cosine) are constructed. The resolution of the secondary table is:

$$
\Delta_{t}=\frac{\Delta_{T}}{N_{t}}=\frac{90^{\circ}}{N_{T} N_{t}}
$$

The angle is approximated as $\theta=n_{T} \Delta_{T}+n_{t} \Delta_{t}$, with $n_{T}$ and $n_{t}$ corresponding to the most and least significant bits, respectively. To calculate the values, the relationship for the sine of a sum of angles is used:

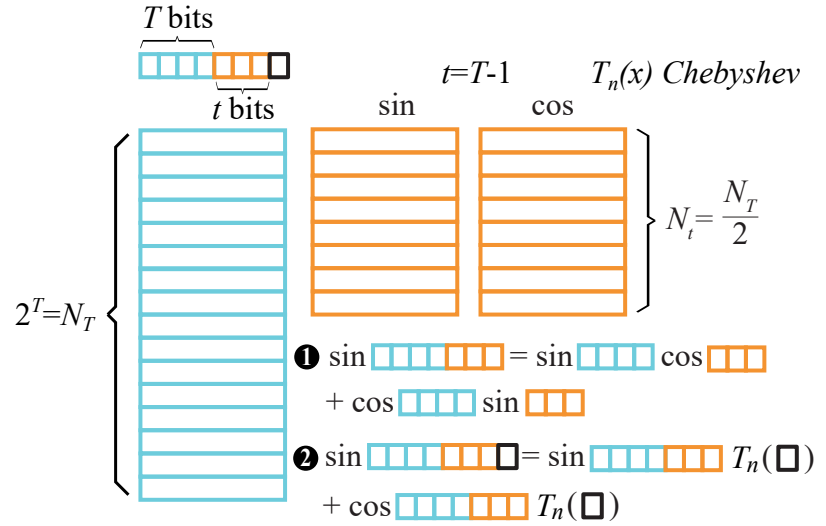

Fig. 4. Procedure for calculation of the $\sin / \cos$ functions.

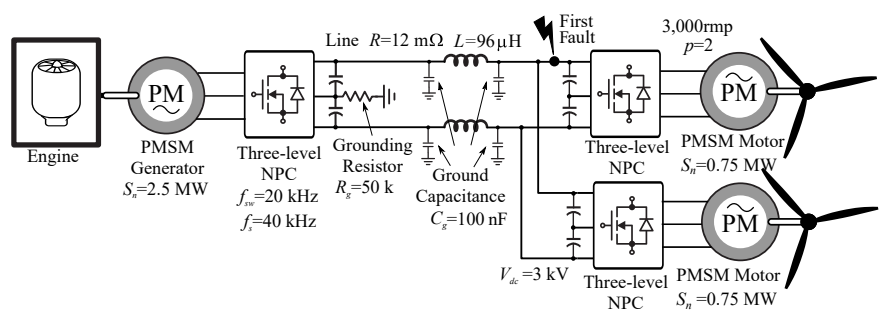

Fig. 5. Proposed architecture for the HEA used in the simulations.

$$
\begin{aligned}
\sin (\theta) \approx \sin \left(n_{T} \Delta_{T}+n_{t} \Delta_{t}\right) & =\sin \left(n_{T} \Delta_{T}\right) \cos \left(n_{t} \Delta_{t}\right) \\
& +\cos \left(n_{T} \Delta_{T}\right) \sin \left(n_{t} \Delta_{t}\right)
\end{aligned}
$$

The total number of entries is $N_{m w}=N_{T}+2 N_{t}$. Considering a fixed number of memory words $N_{m w}$, the relation of $N_{T}$ and $N_{t}$ should minimize $\Delta_{t}$ in (10). By resolving $d \Delta_{t} / N_{t}=0$ with $N_{T}=N_{m w}+2 N_{t}$, the following relationship is obtained:

$$
N_{T}=2 N_{t}=N_{m w} / 2
$$

This leads to an interval $\Delta_{t}=720^{\circ} / N_{m w}^{2}$ instead of $\Delta_{t}=90^{\circ} / N_{m w}$ as it would be required with the single table approach. Once the two-level table procedure has been processed, extra accuracy can be achieved by doing a second iteration $\sin (\alpha+\kappa)=\sin (\alpha) \cos (\kappa)+\cos (\alpha) \sin (\kappa)$ with $\alpha$ representing the higher-order bits and $\kappa$ the lower-order bits. The values $\sin (\alpha)$ and $\cos (\alpha)$ were calculated using the tables and to estimate $\sin (\kappa)$ and $\cos (\kappa)$, the Taylor series could be used $\sin (\kappa) \approx \kappa$ and $\cos (\kappa) \approx 1-\kappa^{2} / 2$. However, the error will be proportional to the angle $\kappa$ and using a Chebyshev approximation with equiripple error for the $[0, \kappa]$ results in more accuracy for the same number of computations; details can be found in [28].

The size of the tables is usually the power of two with $N_{T}=2^{T}$ being $T$ the number of most significant bits and $N_{t}=2^{t}$ being $t=T-1$ the number of least significant bits. The values in these positions ( $T$ bits and $t$ bits) serve as search indexes for the look-up tables. Fig. 4 illustrates the algorithm.

\section{Goertzel Algorithm For the SDFT}

A feature of the SDFT is that it can be expressed iteratively: 


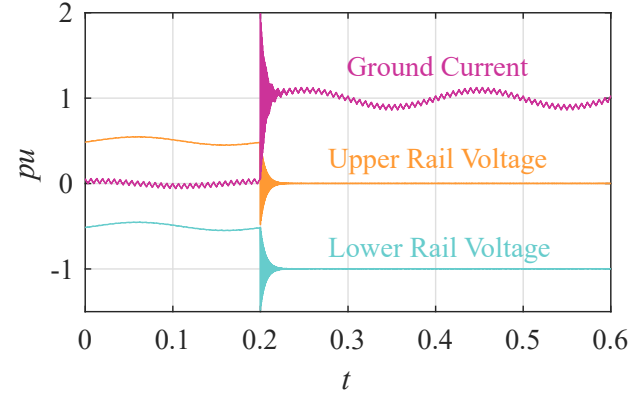

Fig. 6. Simulation results: dc link voltage generator side and ground current.

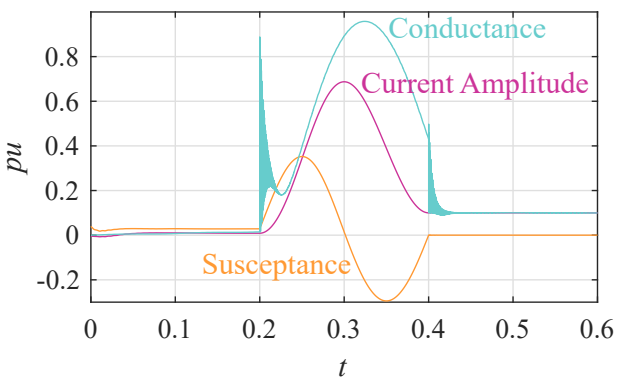

Fig. 7. Simulation results: PIM and toroid magnitudes.

$$
X_{k}[n+1]=e^{j \frac{2 \pi k}{N}} X_{k}[n]+(x[n]-x[n-N])
$$

The algebraic manipulations to obtain this result can be found in [29]. Eq. (13) takes into account the computation delay equal to a sample. Because of the iterative nature of this equation, the next output is only valid if the previous $N$ inputs are also valid and an initialization procedure is needed with zero initial values. As the fundamental component is the only one required to be calculated, (13) results as:

$$
X_{1}[n+1]=e^{j \frac{2 \pi}{N}} X_{1}[n]+(x[n]-x[n-N])
$$

The result only requires pre-calculation of the $e^{j \frac{2 \pi}{N}}$ and it avoids the need to calculate the sine and cosine functions in line, which results in a substantial saving of operations. The disadvantage of this procedure is that the filter is marginally stable and it is necessary to modify the coefficients to avoid having poles outside the unity circle [30], [31] and this reduces the accuracy. The Goertzel configuration shown in Fig. 3(b) [30], [31] only requires the ac current amplitude $\left(\left|I_{1}[n]\right|\right)$ for fault location and hence it is very computationally efficient.

\section{Simulation Results}

Fig. 5 shows the architecture of the HEA selected for simulations using Matlab/Simulink along with all the parameters. The NPC converters connected to the machines use PWM with double update mode (the sampling frequency is twice the switching frequency) [32] and standard vector control [33] with nested dq-current controllers. The NPC converter connected to the PMSM generator regulates the voltage in the DC-distribution. The NPC converters connected to the PMSM

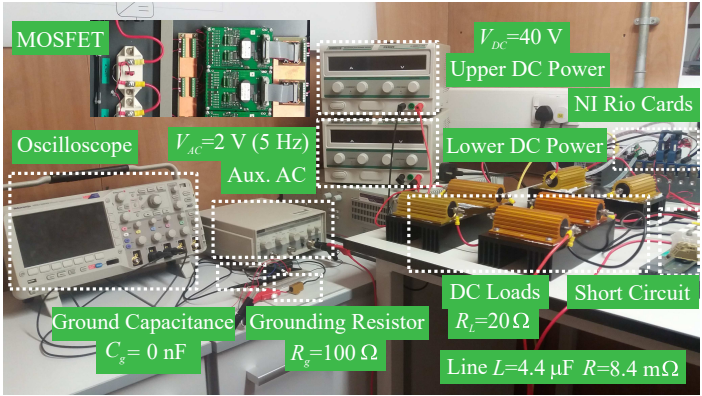

Fig. 8. Set-up for the experimental results.

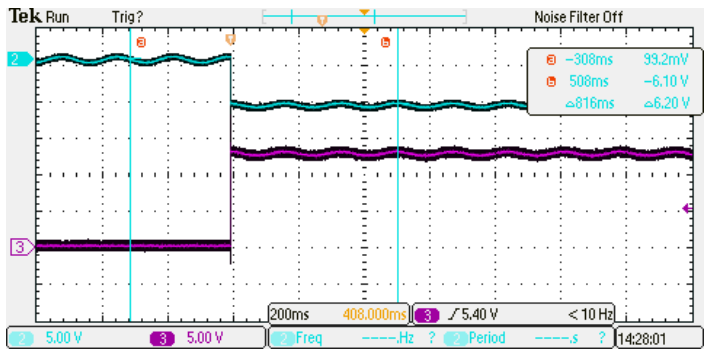

Fig. 9. Experimental results: ground current (cyan) and rail voltage (magenta).

motors have speed regulators. The control for balancing the DC capacitors of the NPC converter is explained in [34]. The ground resistor value was selected as $R_{g}=50 \mathrm{k} \Omega$ to allow 30 $\mathrm{mA}$ circulating for the first-fault. This requires a voltage source of $150 \mathrm{~V}$ ac, 20 times lower than the dc voltage [16]. Fig. 6 shows the voltage to ground of the rails at the generator side. There is a voltage shift from $0.5 \mathrm{pu}$ to $1 \mathrm{pu}$ with an overshoot due to the capacitance to ground. The initial small oscillation is due to the PIM ac source. Fig. 6 also shows the current through the grounding resistor. The initial ac current is due to the substantial capacitance to ground. The light damping is only provided by the parasitic resistance of the DC transmission line (other elements are ideal: switches, no magnetic losses and no ESR in the DC capacitors). In practice, these highfrequency oscillations would be more damped because of the non-modelled additional losses of the system. These highfrequency oscillations are easily filtered out by the underlying MAF filter. After the fault transient, the current presents a dc component (30 mA) and an oscillating component. Fig. 7 shows the proposed sliding DFT that uses an MAF to estimate the conductance as $1 \mathrm{pu}$ after the ground fault corresponding to the short-circuit to ground. The detected susceptance is slightly higher than zero due to the parasitic capacitance. Fig. 7 also shows the Goertzel algorithm being used to estimate the ac current amplitude to detect the fault. This algorithm is implemented in the microcontroller of the toroid located upstream of the ground fault.

\section{EXPERIMENTAL RESULTS}

Fig. 8 shows the set-up for the experiments along with all the parameters. The experimental set-up represents the simplified schematic shown in Fig. 2a. The experimental set-up was scaled to low voltage $(40 \mathrm{~V})$ and had no capacitance to ground 


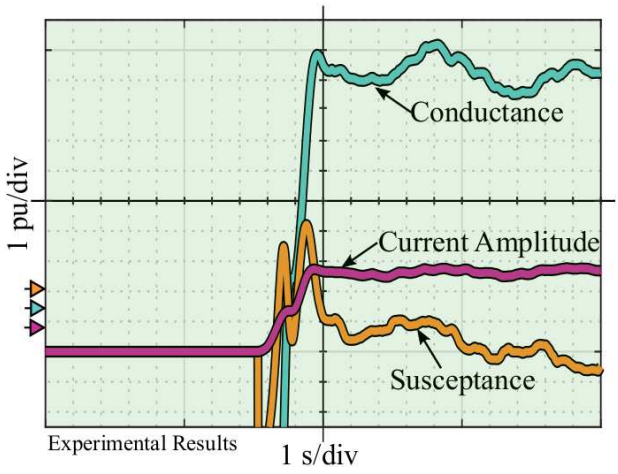

Fig. 10. Experimental results: PIM and toroid magnitudes.

due to limitations in the protection relays. The low-impedance ground-fault was emulated using a MOSFET activated by a NI CompactRIO system. Fig. 9 illustrates the voltage imposed by the auxiliary ac source and the current circulating through the grounding resistor. Initially, the current through the grounding resistor is zero due to the lack of capacitance to ground. After the first-fault in the high rail at $t=0.6 \mathrm{~s}$, the current has a dc component due to the main voltage source and an ac component due to the auxiliary ac source. The offset in the voltage after the fault is due to the internal impedance of the ac source. The current and voltage measurements were captured using a middle-range oscilloscope with precision $3 \%$; the grounding resistor has a standard tolerance of $10 \%$. The measurements corresponding to Fig. 9 were recorded in an Excel file CVS (comma-separated values) generated by the oscilloscope; the sampling frequency was $250 \mathrm{MHz}$. The Excel CVS file with the experimental results was exported to Matlab to be used with the Signal Builder block to feed the same Simulink block used for the PIM implementation in the simulations at a sample rate of $1 \mathrm{kHz}$. Hence, the algorithm can be implemented easily in middle-range DSPs or FPGAs. Fig. 10 shows the results of the PIM detection procedure as shown in Fig. 3 (including an optional bandpass filter). Even with these uncertainties and noisy signals, the proposed algorithm for the PIM as shown in Fig. 3(b) detects the conductance variation. The conductance is initially nil (no fault) and after the short-circuit fault to ground, the conductance rises to around unity (corresponding to the grounding resistors). With no capacitance to ground, the susceptance tends to zero. Fig. 10 also shows the effective detection of the current amplitude using the Goertzel algorithm.

\section{CONCLUSION}

This letter proposes an accurate and computationallyefficient PIM algorithm for IT systems based on the SDFT. The method for first-fault detection is effective in estimating the fault-impedance even with noisy measurements because of the heavy filtering of the MAF implicit in the SDFT. Double look-up tables with optimized size reduce computations in trigonometric functions. The Goertzel algorithm for the SDFT with little computational load may allow the use of low-cost microcontrollers integrated in the toroids for fault location.

\section{REFERENCES}

[1] R. Jansen, C. Bowman, A. Jankovsky, R. Dyson, and J. Felder, "Overview of nasa electrified aircraft propulsion (eap) research for large subsonic transports," in 53rd AIAA/SAE/ASEE Joint Propulsion Conf., A. I. of Aeronautics and Astronautics, Eds., Jul. 2017.

[2] T. Schroter and D. Schulz, "The electrical aircraft network; benefits and drawbacks of modifications," IEEE Trans. Aerospace and Electron. Systems, vol. 49, no. 1, pp. 189-200, Jan 2013.

[3] E. Joffe and K. Lock, Grounds for Grounding: A Circuit to System Handbook. Wiley, 2011.

[4] S. M. Braden, M. J. Doherty, and S. M. Scott, "Current return network," U.S. Patent US8 031 458, 2011.

[5] G. G. Gutierrez, D. M. Romero, M. R. Cabello, E. Pascual-Gil, L. D. Angulo, D. G. Gomez, and S. G. Garcia, "On the design of aircraft electrical structure networks," IEEE Trans. Electromagnetic Compatibility, vol. 58, no. 2, pp. 401-408, April 2016.

[6] F. Jullien and I. Heritier, The IT earthing system (unearthed neutral) in LV. Schneider Electric, June 1999, no. Cahier technique no. 178.

[7] C. Meijer, J. K. van der Ven, and R. Ross, "Emc and electrical safety on board ships how emi filters undermine the protection against electric shock," in 2013 Int. Symp. on Electromagnetic Compatibility, Sept 2013, pp. 1034-1038.

[8] H. Sellner, "Electrical safety for dc systems," Bender GmbH \& Co.KG, Gruenberg, Germany, Tech. Rep., 2013.

[9] M. Hartmann, H. Ertl, and J. W. Kolar, "Emi filter design for a $1 \mathrm{mhz}$, $10 \mathrm{kw}$ three-phase/level pwm rectifier," IEEE Trans. on Power Electron., vol. 26, no. 4, pp. 1192-1204, April 2011.

[10] L. Orozco, "Synchronous detectors facilitate precision low-level measurements," Analog Dialogue, vol. 48, no. 11, Nov. 2014.

[11] A. Oppenheim and R. Schafer, Discrete-time Signal Processing. Prentice Hall, 1989.

[12] C. Abascal, "What's your sine? find the right algorithm for ddfs on a dsp," Embedded systems design, vol. 21, no. 10, pp. 12-21, Oct. 2008.

[13] P. Cheung, "Topic 5: Functions evaluation," Course Slides for E3.05 Digital System Design. Imperial College London, UK., Jan. 2008.

[14] J.-C. Chih, J.-Y. Chou, and S.-G. Chen, "An efficient direct digital frequency synthesizer based on two-level table lookup," in Proc. of the 2001 IEEE Int. Frequncy Control Symp. and PDA Exhibition, 2001, pp. 824-827.

[15] S.-G. Chen, J.-C. Chih, and J.-Y. Chou, "Direct digital frequency synthesis based on a two-level table-lookup scheme," J. of VLSI signal processing systems for signal, image and video technology, vol. 45, no. 3 , pp. 153-160, Dec 2006.

[16] P. Olszowiec, Insulation Measurement and Supervision in Live AC and DC Unearthed Systems. Springer Berlin Heidelberg, 2012.

[17] L. Asiminoaei, R. Teodorescu, F. Blaabjerg, and U. Borup, "Implementation and test of an online embedded grid impedance estimation technique for pv inverters," IEEE Trans. Ind. Electron., vol. 52, no. 4, pp. 11361144, Aug 2005.

[18] R. Pena-Alzola, M. Liserre, F. Blaabjerg, M. Ordonez, and T. Kerekes, "A self-commissioning notch filter for active damping in a three-phase lcl -filter-based grid-tie converter," IEEE Trans. Power Electron., vol. 29, no. 12, pp. 6754-6761, Dec 2014.

[19] F. Paz and M. Ordonez, "High-accuracy impedance detection to improve transient stability in microgrids," IEEE Trans. Ind. Electron., vol. 64, no. 10, pp. 8167-8176, Oct 2017.

[20] M. Meade, Lock-in Amplifiers: Principles and Applications, ser. IEE electrical measurement series. P. Peregrinus, 1983.

[21] R. Pena-Alzola, D. Campos-Gaona, P. F. Ksiazek, and M. Ordonez, "Dclink control filtering options for torque ripple reduction in low-power wind turbines," IEEE Trans. Power Electron., vol. 32, no. 6, pp. 48124826, June 2017.

[22] F. Paz and M. Ordonez, "An embedded impedance measurement for dc microgrids based on a lock-in amplifier," in 2016 IEEE 7th Int. Symp. on Power Electron. for Distributed Generation Systems (PEDG), 2016, pp. $1-6$.

[23] F. Paz and M. Ordonez, "Embedded fault location in dc microgrid systems based on a lock-in amplifier," in 2017 IEEE 8th Int. Symp. on Power Electron. for Distributed Generation Systems (PEDG), April 2017, pp. 1-6.

[24] F. Paz and M. Ordonez, "An impedance-based islanding detection method for dc grids," in 2018 9th IEEE Int. Symp. on Power Electron. for Distributed Generation Systems (PEDG), 2018, pp. 1-7.

[25] - "High-impedance fault detection method for dc microgrids," in 2019 IEEE 10th Int. Symp. on Power Electron. for Distributed Generation Systems (PEDG), 2019, pp. 787-792. 
[26] — "Fast and efficient solar incremental conductance mppt using lock-in amplifier," in 2015 IEEE 6th Int. Symp. on Power Electron. for Distributed Generation Systems (PEDG), 2015, pp. 1-6.

[27] — , "High-performance solar mppt using switching ripple identification based on a lock-in amplifier," IEEE Trans. Ind. Electron., vol. 63, no. 6, pp. 3595-3604, 2016.

[28] W. Press, Numerical Recipes in C: The Art of Scientific Computing, ser. Numerical recipes in FORTRAN. Cambridge University Press, 1992.

[29] I. Hickman, Electronic Circuits, Systems and Standards: The Best of $E D N$, ser. EDN series for design engineers. Elsevier Science, 2016.

[30] E. Jacobsen and R. Lyons, "The sliding dft," IEEE Signal Processing Mag., vol. 20, no. 2, pp. 74-80, Mar 2003.

[31] - "An update to the sliding dft," IEEE Signal Processing Mag., vol. 21, no. 1, pp. 110-111, Jan 2004.

[32] V. Blasko, V. Kaura, and W. Niewiadomski, "Sampling of discontinuous voltage and current signals in electrical drives: a system approach," IEEE Trans. Ind. Applicat., vol. 34, no. 5, pp. 1123-1130, 1998.

[33] W. Leonhard, Control of Electrical Drives. Springer Berlin Heidelberg, 2012.

[34] A. Yazdani and R. Iravani, Voltage-Sourced Converters in Power Systems: Modeling, Control, and Applications, ser. Wiley - IEEE. John Wiley and Sons, 2010. 